\section{Professor Hans Merk zum 60. Geburtstag}

\author{
Am 29. November feierte Prof. Dr. Hans F. Merk, Aachen, seinen \\ 60. Geburtstag. Der Ärzteverband Deutscher Allergologen gratuliert \\ seinem gerade frisch ins Amt gekommenen Präsidenten.
}

$\mathrm{H}$ ans Merk wurde am 29. November 1949 in Wuppertal geboren. Er studierte Humanmedizin in Düsseldorf, wo er am Institut für Pharmakologie auch promovierte. Nach seiner wissenschaftlichen Assistenzzeit an der Hautklinik der Universität Düsseldorf wechselte er nach Köln, wo er bereits im Jahre 1983 den Titel Facharzt für Dermatologie und Venerologie erwarb und noch im selben Jahr zum Oberarzt der Hautklinik der Universität zu Köln aufstieg. Konsequent verfolgte er seinen wissenschaftlichen Weg und vollendete in ungewöhnlich kurzer Zeit seine Habilitationsarbeit, weshalb ihm schon 1984 die Venia legendi in Dermatologie und Venerologie in Köln verliehen wurde.

Wiederum wenige Jahre später erfolgte in Köln 1987 die Ernennung zum Universitätsprofessor (C3) für Dermatologie und Venerologie. Merks besonderes Interesse gehörte schon zu dieser Zeit der Dermatopharmakologie und -toxikologie und den Arzneimittelreaktionen an der Haut. Im Jahre 1994 wurde er zum C4Professor für Dermatologie an die Medizinische Fakultät der RWTH Aachen und zum Direktor der Klinik für Dermatologie und Allergologie des Universitätsklinikums Aachen berufen. Diese Klinik verfolgt seither in besonderer Weise die von Hans Merk vorgegebenen klinischen Schwerpunkte, zu denen die Allergologie, die allgemeine Dermatologie und Venerologie, die allergische Kontaktdermatitis, die Photodermatosen und die Urtikaria gehören.

\section{Ausgezeichneter Forscher und begabter Brückenbauer}

Zahlreiche Auszeichnungen und Preise zeugen von der hohen Qualität seines wissenschaftlichen Wirkens - so der Homburg-Preis 1981, der Oscar-GansFörderpreis 1985 und der Herbert-Herx-

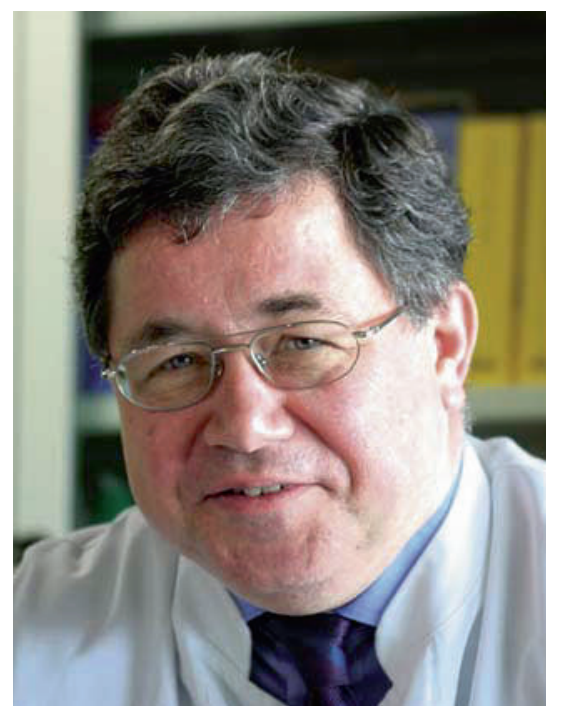

Ad multos annos: Hans F. Merk

heimer-Förderpreis der Deutschen Gesellschaft für Allergie- und Immunitätsforschung 1992. Hans Merk ist Vorsitzender des Habilitationsausschusses der Medizinischen Fakultät der RWTH Aachen. Im Jahre 2009 wurde er zudem zum außerordentlichen Mitglied der Arzneimittelkommission der deutschen Ärzteschaft ernannt.

Hans Merk prägte in den vergangenen Jahrzehnten die Allergologie und Dermatologie sowohl in Deutschland als auch international auf vielen Gebieten entscheidend mit. Wichtige wissenschaftliche Forschungsgebiete seiner Klinik betreffen die Immuntoxikologie kleinmolekularer Substanzen, die Pathophysiologie der allergischen Kontaktdermatitis und von unerwünschten Arzneimittelreaktionen der Haut, den Einfluss des extrahepatischen Fremdstoffmetabolismus auf die Sensibilisierung durch kleinmolekulare Substanzen (Kontaktallergene, Arzneimittel) und die Charakterisierung beteiligter Entzündungszellen.
Von 2006 bis 2009 war Merk Vizepräsident des Ärzteverbandes Deutscher Allergologen (ÄDA) und hat in dieser Eigenschaft wesentlich zur positiven Kommunikation zwischen niedergelassenen Allergologen und Klinikern, aber auch zwischen den allergologischen Gesellschaften untereinander beigetragen. Der Erste Gemeinsame Deutsche Allergiekongress der drei deutschen allergologischen Fachgesellschaften 2004 in Aachen wurde von ihm mitgetragen und -organisiert. In diesem Jahr nun machte er dem gesamten ÄDA-Vorstand die Freude, trotz seiner vielfältigen Aufgaben auch das Amt des Präsidenten des ÄDA zu übernehmen.

Hans Merk ist aktuell einer der Herausgeber der im Springer-Verlag erscheinenden Zeitschrift „Der Hautarzt“.

\section{Kölsche un Öcher Jung}

Hans Merk ist bei aller nationalen und internationalen Anerkennung, trotz vieler Reisen und Kongresse im In- und Ausland immer ein Kind des Rheinlandes geblieben und hat in überaus sympathischer Weise seine regionale Identität gewahrt. Daher möchte ich als Ex-Öcher Dir, lieber Hans, der Du Kölsche un Öcher Jong bist, einige Wünsche mit auf den Weg geben: „Leeve Hans, mach et joot, bliev wie do bist un geniess de Zigg. Et es wie et es un et kütt wie et kütt, ävver, et hät noch immer jot jejange!"

Damit es der Allergologie auch in Zukunft gut gehen wird, sei uns allen Deine tatkräftige, konsequente, aber auch diplomatische Art noch lange Zeit vergönnt.

Prof. Dr. Ludger Klimek, Wiesbaden

Impressum „Der ÅDA informiert“
Verantwortlich für den Inhalt
Prof. Dr. Ludger Klimek, Wiesbaden
Redaktion
Markus Seidl
Verlag
@ Urban \& Vogel GmbH, München
ÄDA-Geschäftsstelle
Service Systems, Dreieich
Tel.: (o 61 03) 62273
Fax: (o 61 03) 697019
E-Mail: info@aeda.de
Internet: www.aeda.de

Verantwortlich für den Inhalt Prof. Dr. Ludger Klimek, Wiesbaden

\section{Redaktion}

Markus Seidl

\section{Verlag}

ÄDA-Geschäftsstelle

Service Systems, Dreieich

(o 61 O3) 62273

E-Mail:infor

Internet: www.aeda.de 